\title{
Fuzzy C-means Clustering for 3D Seismic Parameters Processing
}

\author{
Fuqun Zhao \& Liang Le \\ School of Education and Science, Xian'yang Teachers University \\ Xian’yang 712000, China \\ E-mail: fuqunzhao@126.com
}

Fund Project: Special scientific research funded projects in 2006 of Xian'yang Teachers University (Project Number:07XSYK224)

\begin{abstract}
3D seismic parameters can reflect the features of petroleum reservoir from different profiles. By analizing the 3D seismic parameters, we can assess the parameters of the reservoir characterization, such as deposition, structure and growth history, fluid saturation and so on. The traditional clustering methods can't capture the degree of similarity between reservoir parameters very well, so we introduced in this paper the application of fuzzy C-means (FCM) clustering for the processing of 3D seismic parameters. It begins with the analizing the relationship between 3D seismic parameters and reservoir characterization parameters, and then we process the 3D seismic parameters with FCM and assess the parameters of reservoir characterization. The testing results show that FCM can classify the 3D parameters more accurately and provide a good evidence for the research of petroleum reservoir.
\end{abstract}

Keywords: 3D seismic parameters, Structure and growth history, Fuzzy C-means clustering

\section{Introduction}

Fuzzy C-means (FCM) clustering is one of the essential branches of non-supervisory pattern and it was widely used in pattern recognition, data mining, computer vision, as well as in areas such as fuzzy control. Now, FCM has become a relatively mature technology. In the field of petroleum engineering, it has been widely applied to the evaluation of reservoir quality (Chen, Liang, 1997), reservoir classification, Petroleum exploration and decision-making (Zhu, Kejun, 1999) and so on.

In this paper, we introduced FCM to deal with 3D seismic parameters so as to assess some of the parameters of reservoir characterization, such as structure and growth history, permeability, fluid saturation, and master their distribution. First, we introduced the principle of FCM; then we use FCM to deal with three-dimensional seismic parameters; lastly, we make a summary.

\section{The Principle of Fuzzy C-Means Clustering}

Fuzzy C-Means (FCM) is a method of clustering which allows one piece of data to belong to two or more clusters. This method is frequently used in pattern recognition. It is based on minimization of the following objective function: 


$$
J_{m}=\sum_{i=1}^{N} \sum_{j=1}^{C} u_{i j}^{m} \square x_{i}-c_{j} \square^{2}
$$

Where $m$ is any real number greater than 1 , it was set to 2.00 by Bezdek; $u_{i j}$ is the degree of membership of $x_{i}$ in the cluster $j ; x_{i}$ is the $i^{t h}$ of d-dimensional measured data $; c_{j}$ is the d-dimension center of the cluster and $\left\|^{*}\right\|$ is any norm expressing the similarity between any measured data and the center.

Fuzzy partitioning is carried out through an iterative optimization of the objective function shown above, with the update of membership $u_{i j}$ and the $c_{j}$ cluster centers by:

$$
\begin{gathered}
u_{i j}=\frac{1}{\sum_{k=1}^{c}\left(\frac{\square x_{i}-c_{j} \square}{\square x_{i}-c_{k} \square}\right)^{\frac{2}{m-1}}} \\
c=\frac{\sum_{i=1}^{N} u_{i j}^{m} x_{i}}{\sum_{i=1}^{N} u_{i j}^{m}}
\end{gathered}
$$

This iteration will stop when

$$
\max \left\{\left|u_{i j}^{k+1}-u_{i j}^{k}\right|\right\}<\varepsilon
$$

Where $\varepsilon$ is a termination criterion between 0 and 1 and $\mathrm{k}$ are the iteration steps.

This procedure converges to a local minimum or a saddle point of $J_{m}$.

The algorithm is composed of the following steps:

1. Initialize $U=\left[u_{i j}\right]$ matrix, $U(0)$

2. At k-step: calculate the centers vectors $C(\mathrm{k})=\left[c_{j}\right]$ with $U(\mathrm{k})$

$$
c=\frac{\sum_{i=1}^{N} u_{i j}^{m} x_{i}}{\sum_{i=1}^{N} u_{i j}^{m}}
$$

3. Update $\mathrm{U}(\mathrm{k}), \mathrm{U}(\mathrm{k}+1)$

$$
u_{i j}=\frac{1}{\sum_{k=1}^{c}\left(\frac{\square x_{i}-c_{j} \square}{\square x_{i}-c_{k} \square}\right)^{\frac{2}{m-1}}}
$$

If $\|U(k+1)-U(k)\|<\varepsilon$ then STOP; otherwise return to step 2.

\section{FCM for 3d Seismic Parameters Processing}

In the 3D Seismic Parameters Processing, we first import a potentially large number of independent data (such as seismic amplitude), and quickly assess which are most related to the dependent data (such as porosity-thickness) with FCM, then produce a cluster pattern map of those most-related independent data or 
any user selected data contained in the imported file (correlation and ranking is provided as output). The FCM is very robust as it works well in cases where the dependent data (well control) population is small. In addition to producing a cluster map, an output file can be generated that contains grid location(x,y), cluster rank and cluster mean-value from the dependent data.

Here, we give you an example. The data in table 1 is a portion of input data used for testing. It was used as input data. In the table, the rows $\mathrm{A}$ and $\mathrm{B}$ are the coordinates, row $\mathrm{C}$ is numeric well identifier, the row $\mathrm{D}$ and $\mathrm{E}$ are well information, those are dependent data. Rows from $\mathrm{F}$ to $\mathrm{L}$ are independent data, those are seismic time information.

The figure 1 is the clustering results map with FCM, and it contains four parts: the coordinates of the independent data $(\mathrm{x}, \mathrm{y})$, the clustering map, the cluster groups and the clustering ranks. These cluster groups represent areas of similar structural growth history. The thinnest areas, with maximum growth, are ranked as 1 , here is cluster 3 on the map, the quality of the reservoir is the best. The thickest areas, with minimum growth, are ranked as 3 , here is cluster 2 on the map, the quality of the reservoir is the worst. The quality and the growth history of the reservoir ranked 2 are between cluster 2 and cluster 3.

\section{Conclusion}

The purpose of FCM is to find a reasonable classification system for a given sample with mathematical method, and solve the classification problem in the reservoir description. In this paper, we introduce fuzzy C-means clustering method to the study of dealing with three-dimensional seismic parameters. The experiment results show that, it can deal with the three-dimensional seismic parameters effectively, so we can analize some of the reservoir characterization parameters, master the distribution of the reservoir caracterization parameters, and improve the exploration and development effectiveness of the oil and gas reservoir.

\section{References}

Fan, Ji-zong. (2002). Cluster Analysis in Reservoir Classification. Inner Mongolia Petrochemical, (29):124 126.

Gao, Xin-bo. (2004). Fuzzy Cluster Analysis and Its Applications. Xidian University, 1 5, 6 28.

Mark A.Sippel. (2001). Intelligent Computing System for Reservoir Analysis and Risk Assessment of the Red River Formation.

Mohanad Alata, Mohammad Molhim, and Abdullah Ramini. (2008). Optimizing of Fuzzy C-Means Clustering Algorithm Using GA. Proceedings of World Academy of Science. Engineering and Technology, 29(5): 224 229.

M. M. Saggaf \& Ed L. Nebrija. (2003). A fuzzy logic approach for the estimation of facies from wire-line logs. The American Association of Petroleum Geologists, 87(7):1223 1240. 
Table 1. Portion of Input Data Used for Testing

\begin{tabular}{|l|l|l|l|l|l|l|l|l|l|l|l|l|}
\hline & A & B & C & D & E & F & G & H & I & J & K & L \\
\hline 1 & 1212821 & 149427 & $3.3 \mathrm{E}+09$ & -6066.8 & -1747.3 & 652 & 991 & 1120 & 1554 & 1618 & 1713 & 1805 \\
\hline 2 & 1212474 & 145067 & $3.3 \mathrm{E}+09$ & -6059.4 & -1750.7 & 645 & 989 & 1118 & 1546 & 1612 & 1707 & 1799 \\
\hline 3 & 1211646 & 144209 & $3.3 \mathrm{E}+09$ & -6052 & -1752 & 642 & 993 & 1118 & 1548 & 1613 & 1708 & 1799 \\
\hline 4 & 1212374 & 144767 & $3.3 \mathrm{E}+09$ & -6052.4 & -1753 & 643 & 990 & 1117 & 1547 & 1612 & 1707 & 1798 \\
\hline 5 & 1215767 & 142373 & $3.3 \mathrm{~F}+09$ & -6060.4 & -1757.3 & 652 & 997 & 1126 & 1553 & 1615 & 1713 & 1801 \\
\hline 6 & 1214184 & 150850 & $3.3 \mathrm{E}+09$ & & -1759.1 & 660 & 996 & 1125 & 1561 & 1623 & 1713 & 1807 \\
\hline 7 & 1207014 & 152537 & $3.3 \mathrm{E}+09$ & -6119.93 & -1762.45 & 643 & 984 & 1117 & 1551 & 1614 & 1703 & 1803 \\
\hline 8 & 1216262 & 152792 & 95 & -6184.9 & -1831 & 666 & 1005 & 1129 & 1568 & 1635 & 1783 & 1831 \\
\hline 9 & 1207727 & 148819 & 1555 & & & 635 & 984 & 1112 & 1545 & 1612 & 1708 & 1803 \\
\hline 10 & 1206414 & 148488 & 1554 & & & 636 & 983 & 1115 & 1541 & 1610 & 1710 & 1803 \\
\hline 11 & 1209040 & 148488 & 1553 & & & 636 & 982 & 1108 & 1554 & 1613 & 1708 & 1801 \\
\hline 12 & 1206414 & 148819 & 1552 & & & 636 & 984 & 1116 & 1544 & 1613 & 1712 & 1804 \\
\hline 13 & 1704742 & 148488 & 1551 & & & 636 & 982 & 1114 & 1593 & 1609 & 1709 & 1803 \\
\hline 14 & 1207070 & 149482 & 1550 & & & 637 & 987 & 1118 & 1551 & 1617 & 1715 & 1805 \\
\hline 15 & 1208055 & 148819 & 1549 & & & 637 & 983 & 1113 & 1548 & 1614 & 1710 & 1802 \\
\hline 16 & 1210025 & 144847 & 1548 & & & 637 & 988 & 1119 & 1552 & 1613 & 1706 & 1800 \\
\hline
\end{tabular}

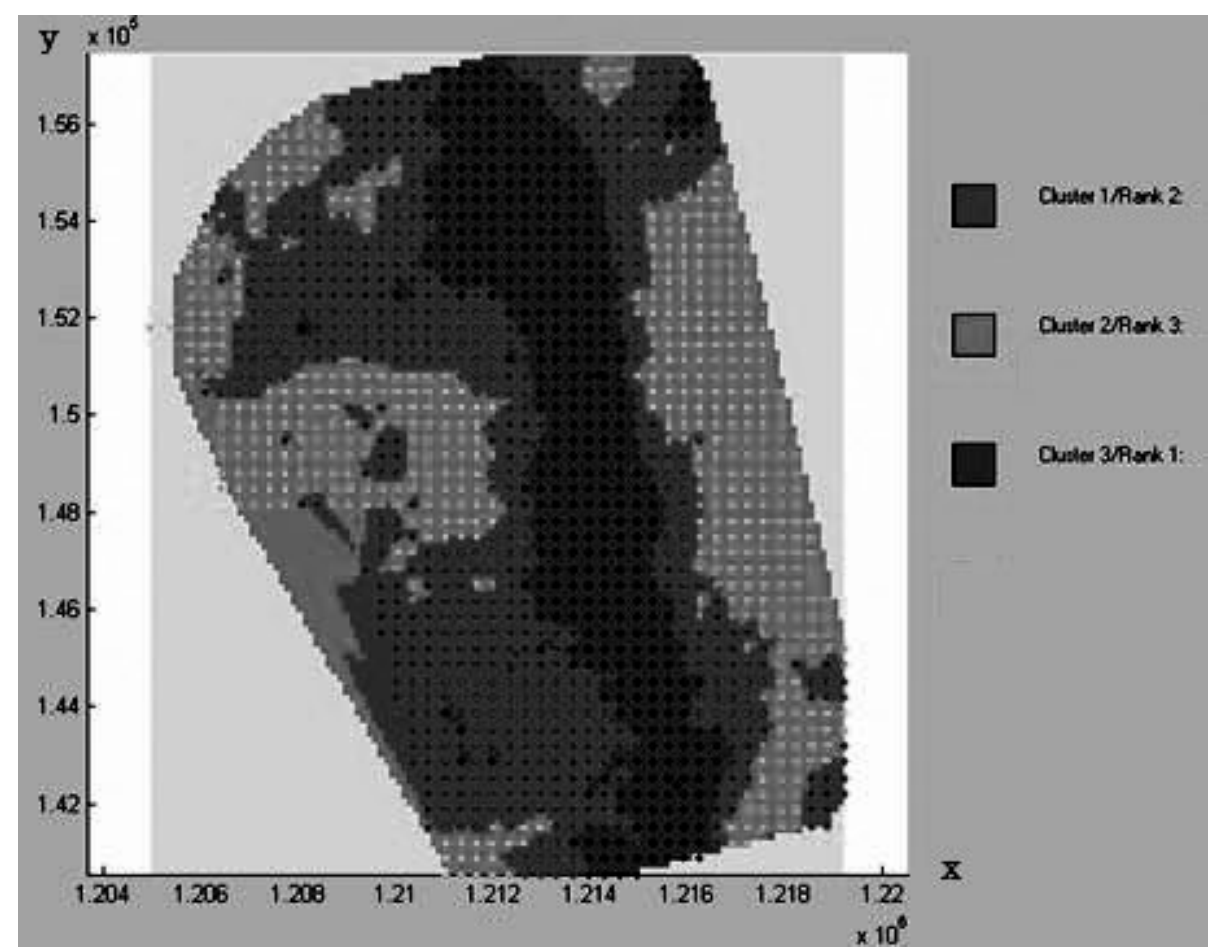

Figure 1. The Clustering Results 